\title{
Ingestão de lipídios na dieta e indicadores antropométricos de adiposidade em policiais militares
}

\author{
Dietary fats and measures of adiposity \\ in military policemen
}

Cristina DONADUSSI ${ }^{1}$

Ana Flávia OLIVEIRA²

Elis Carolina de Souza FATEL ${ }^{3}$

Jane Bandeira DICHI ${ }^{4}$

Isaias DICHI ${ }^{4}$

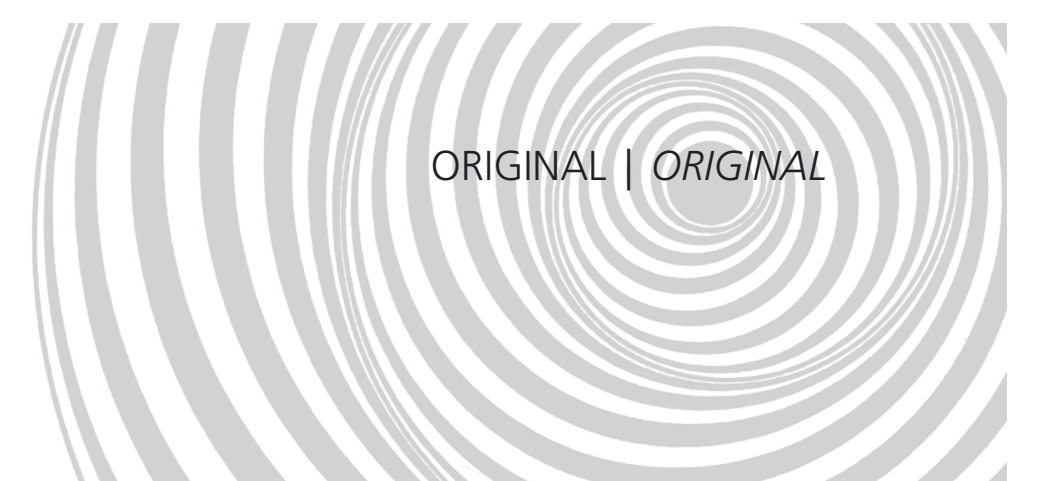

(5)

RES U M O

\section{Objetivo}

Investigar se há relação entre a ingestão de lipídios e os indicadores antropométricos de adiposidade em policiais militares do Município de Cascavel, (PR).

\section{Métodos}

A amostra constituiu-se de 183 policiais militares do sexo masculino do $6^{\circ}$ Batalhão da Polícia Militar de Cascavel (PR), com idade entre 21 e 50 anos. Avaliou-se o peso, a estatura, o índice de massa corporal, a circunferência abdominal e a ingestão alimentar, por meio do recordatório de 24horas. Relacionaram-se os resultados de ingestão de lipídios da dieta com os indicadores antropométricos índice de massa corporal, circunferência abdominal e percentual de gordura corporal.

\section{Resultados}

O diagnóstico nutricional mais freqüente em 117 (63,9\%) dos policiais, segundo o índice de massa corporal, foi de sobrepeso e obesidade; 22 (12,0\%) apresentaram risco elevado para complicações metabólicas associadas à obesidade pelo indicador circunferência abdominal e 26 (18,6\%) elevado percentual de gordura corporal. A ingestão de lipídios se mostrou aumentada em 53 (70,7\%) dos 75 inquéritos validados, no entanto apenas $24(32,0 \%)$ ingeriam lipídios saturados acima da recomendação. Houve correlação positiva apenas entre a ingestão de lipídios totais e o indicador antropométrico de adiposidade circunferência abdominal $(p=0,03)$.

\footnotetext{
${ }^{1}$ Universidade Estadual de Londrina, Programa Pós-Graduação, Centro de Ciências da Saúde. Av. Roberto Koch, 60, Cervejaria, 86038-440, Londrina, PR, Brasil. Correspondência para/Correspondence to: C. DONADUSSI. E-mail: <cristinadonadussi@hotmail.com>.

${ }^{2}$ Universidade Tecnológica Federal do Paraná. Londrina, PR, Brasil.

3 Faculdade Assis Gurgacz. Cascavel, PR, Brasil.

${ }^{4}$ Universidade Estadual de Londrina, Departamento de Clínica Médica, Centro de Ciências da Saúde. Londrina, PR, Brasil.
} 
848 | C. DONADUSSI et al.

\section{Conclusão}

Este estudo sugere que a ingestão de lipídios acima das recomendações pode ser um fator relacionado à adiposidade abdominal na população estudada.

Termos de indexação: Gordura abdominal. Lipídios na dieta. Militares. Obesidade.

\section{A B S T R A C T}

\section{Objective}

The objective of this study was to investigate whether there is any relationship between dietary fats and measures of adiposity in military policemen from Cascavel (PR), Brazil.

\section{Methods}

The study evaluated 183 male military policemen from the $6^{\text {th }}$. Military Police Force of Cascavel (PR), aged from 21 to 50 years. Weight, height, body mass index and waist circumference were measured and food intake was determined by the 24-hour dietary recall. Dietary fats were related with the anthropometric indicators body mass index, waist circumference and percentage of body fat.

\section{Results}

The most common nutritional diagnoses found in 117 (63.9\%) of the policemen were overweight and obesity. Waist circumference showed that $22(12.0 \%)$ of these men were at high risk of metabolic complications associated with obesity and 26 (18.6\%) had high body fat percentiles. Fat intake was high in 53 (70.7\%) of the 75 validated surveys, however, only 24 (32.0\%) ingested saturated fats above the recommended levels. There was a positive correlation only between total fat intake and the anthropometric indicator waist circumference $(p=0.03)$.

\section{Conclusion}

This study suggests that fat intake above the recommended levels may be associated with abdominal adiposity in this population.

Indexing terms: Abdominal fat. Dietary fats. Military personnel. Obesity.

\section{N T R O D U Ç Ã O}

O papel da nutrição expandiu-se significativamente e, atualmente, é visto como ferramenta que pode ser utilizada na defesa contra as doenças crônicas não-transmissíveis ${ }^{1}$. O estado nutricional é o resultado do equilíbrio entre o consumo de nutrientes e o gasto energético do organismo para suprir as necessidades nutricionais, sendo que a avaliação nutricional visa a identificar problemas nutricionais, possibilitando a promoção ou recuperação do estado de saúde do indivíduo².

A transição nutricional tem se caracterizado pela redução da prevalência da desnutrição e pelo aumento generalizado de sobrepeso, que se destaca como um dos maiores problemas atuais de saúde pública ${ }^{3,4}$. Dados da Pesquisa de Orçamento Familiar revelam que o excesso de peso na população brasileira afeta $41,1 \%$ dos homens e
$40,0 \%$ das mulheres, sendo que, desse grupo, a obesidade atinge 8,9\% dos homens e 13,1\% das mulheres adultas 5 .

O sobrepeso e a obesidade são desequilíbrios de peso geralmente analisados pelo indicador Índice de Massa Corporal (IMC). No entanto, a medida isolada da Circunferência Abdominal (CA) é importante, pois prediz com grande confiabilidade o tecido adiposo visceral e está mais bem relacionada com as doenças cardiovasculares ateroscleróticas ${ }^{6}$.

Embora a obesidade se mostre como fator de risco independente para doença cardiovascular, é importante ressaltar a forte associação entre obesidade, hipertensão arterial e dislipidemias ${ }^{3}$. Estudos epidemiológicos sugerem que, além do consumo de gorduras saturadas, outros fatores como dietas ricas em energia, colesterol e bebida alcoólica também se relacionam positivamente com estas doenças ${ }^{7}$. 
Não é de conhecimento, até o momento, qualquer trabalho nacional publicado que tenha avaliado as condições de saúde de policiais militares, principalmente em relação ao seu estado nutricional e à alimentação. O ritmo diário de trabalho e a responsabilidade perante a sociedade podem levar estes indivíduos a situações de estresse que, aliadas à má alimentação e à inatividade física, podem ocasionar o desenvolvimento de doenças cardiovasculares.

Portanto, o objetivo deste estudo foi investigar se há relação entre a ingestão de lipídios com os indicadores antropométricos de adiposidade em policiais militares do Município de Cascavel (PR).

\section{MÉ T O D O S}

Trata-se de um estudo descritivo e transversal, que avaliou 183 policiais do sexo masculino, pertencentes ao $6^{\circ}$ Batalhão da Polícia Militar ( $6^{\circ}$ BPM) da cidade de Cascavel (PR). O presente estudo faz parte do Projeto de Extensão denominado "Programa de Educação em Nutrição: Acompanhamento de Militares do Exército e da Polícia do Oeste do Paraná".

Foram incluídos os policiais militares que trabalhavam há mais de dois anos na corporação e que aceitaram voluntariamente participar da pesquisa. A coleta de dados foi realizada no período de março a abril de 2006 . Todos os participantes assinaram o Termo de Consentimento Livre e Esclarecido. Este projeto foi aprovado pelo Comitê de Ética em Pesquisa, da Universidade Estadual do Oeste do Paraná (Unioeste) (parecer n 185/ 2005).

Os policiais militares foram submetidos à entrevista individual para conhecimento de dados pessoais, como idade, tempo de serviço, atividade desenvolvida na instituição, ingestão alimentar e indicadores antropométricos de adiposidade.

Os indicadores antropométricos de adiposidade estudados foram: índice de massa corporal, circunferência abdominal e percentual de gordura corporal. Durante a realização da antropometria, os indivíduos permaneceram em posição ereta, sem camisa, sem farda, sem sapatos, com o abdômen relaxado, braços estendidos ao longo do corpo e pés juntos. A verificação do peso corpóreo foi feita utilizando-se balança digital da marca Filizola ${ }^{\circledR}$, com capacidade para $150 \mathrm{~kg}$ e variação de $0,1 \mathrm{~kg}$. A verificação da estatura foi realizada mediante utilização de fita métrica inelástica de $150 \mathrm{~cm}$ de comprimento, afixada em parede plana, sem rodapé, a $50 \mathrm{~cm}$ do chão. A partir da verificação do peso e da estatura, calculou-se o Índice de Massa Corporal (IMC; $\left.\mathrm{kg} / \mathrm{m}^{2}\right)$, mediante divisão do peso corpóreo $(\mathrm{kg})$ pela estatura $\left(\mathrm{m}^{2}\right)$.

Para classificação do IMC utilizou-se o critério proposto pela Organização Mundial da Saúde ${ }^{8}$, que estabelece os seguintes limites de corte e seus respectivos diagnósticos nutricionais: Eutrofia de 18,5 a $24,9 \mathrm{~kg} / \mathrm{m}^{2}$; Sobrepeso de 25,0 a $29,9 \mathrm{~kg} / \mathrm{m}^{2}$; Obesidade grau I de 30,0 a $34,9 \mathrm{~kg} / \mathrm{m}^{2}$; Obesidade grau II de 35 a 39,9kg/m²; Obesidade grau III $\geq 40,0 \mathrm{~kg} / \mathrm{m}^{2}$.

Para verificação da circunferência abdominal utilizou-se fita métrica inelástica de $150 \mathrm{~cm}$, sendo a medida realizada no ponto médio entre a crista ilíaca e o rebordo costal inferior ${ }^{9}$. Os valores de circunferência abdominal $\geq 102 \mathrm{~cm}$ foram considerados de risco muito elevado para complicações metabólicas associadas à obesidade ${ }^{10,11}$.

O percentual de gordura corporal foi analisado mediante Bioimpedância Elétrica (BIA) com freqüência de $50 \mathrm{kHz}$, seguindo as recomendações prévias do fabricante (Bodystat ${ }^{\circledR}$ modelo 1500 tetrapolar). O risco de doenças associadas à obesidade foi considerado quando o percentual de gordura corpórea foi maior ou igual a $25 \%^{12}$. Dos 183 participantes, 150 (82\%) fizeram o preparo adequado para a realização deste exame.

O método utilizado para definir o consumo alimentar dos policiais militares foi o recordatório de 24 horas $^{13}$. Para facilitar o processo, utilizou-se um álbum contendo fotos dos alimentos com porcionamentos diferenciados para identificar o tamanho das porções, assegurando, dessa maneira, a melhoria da qualidade da informação. Para mi- 
nimizar a variabilidade intrapessoal, o recordatório foi aplicado em dois momentos consecutivos e a média dos dois valores foi utilizada para análise. As entrevistas foram realizadas em períodos entre terças e sextas feiras, excluindo, também, o dia seguinte a feriados.

Os cálculos da ingestão energética e lipídica de cada indivíduo foram realizados com o auxílio do programa Sistema de Apoio à Decisão em Nutrição (versão 2,5a) ${ }^{14}$. O Gasto Energético Basal (GEB) foi realizado a partir da equação proposta pela Dietary Reference Intake (DRI) ${ }^{15}$, sendo: GEB (Kcal/dia) = 293 - 3,8 $x$ idade (anos) $+456,4 \times$ estatura $(\mathrm{m})+10,12 \times$ peso $(\mathrm{kg})^{16}$.

Com o objetivo de minimizar erros quanto aos dados de ingestão alimentar, foi avaliada a presença de sub-relato ${ }^{17}$. O sub-relato de ingestão alimentar pode ser composto do sub-registro e/ou do subconsumo. O sub-registro é o não-relato de alimentos de fato consumidos, enquanto o subconsumo é a diminuição do consumo alimentar causada pelo próprio instrumento de avaliação. Este dado é medido pela relação entre a ingestão energética (referida) e o gasto energético basal (calculado). Dessa forma, resultados inferiores a 1,3 foram desconsiderados. Assim, apenas 75 (41\%) inquéritos foram validados.

A dieta foi considerada hiperlipídica quando a energia proveniente de lipídios totais era superior a 35\% ${ }^{15}$. Quanto aos lipídios saturados, foi considerada inadequada ingestão maior que $10 \%{ }^{18}$.
Utilizou-se estatística descritiva para apresentação dos dados coletados e a correlação de Spearman para verificar associação entre as variáveis: ingestão de lipídios, índice de massa corporal, circunferência abdominal e percentual de gordura corporal. Os dados foram analisados com o programa estatístico Statistica for Windows, Stat Soft. Fixou-se o nível de rejeição da hipótese de nulidade em 0,05 ( $p \leq 5 \%$ ).

\section{RE S U L T A DOS}

Dentre os policiais avaliados, $146(81,6 \%)$ desenvolvem atividades operacionais e 33 (18,4\%) desenvolvem funções administrativas. A média de idade foi de 35,4 anos (Desvio-Padrão $\mathrm{DP}=6,3)$, variando entre 21 a 50 anos, sendo a mediana de 35 anos.

Estimativas quanto à média e à mediana dos indicadores antropométricos e da ingestão alimentar encontram-se na Tabela 1 . O gasto energético basal dos policiais militares avaliados foi de 1738 (DP=118,2kcal), enquanto que a ingestão energética média relatada foi de 2230 (DP=812kcal). Observou-se alto consumo energético de calorias provenientes de lipídios totais, cuja média de ingestão foi de 39,3\%, sendo o ideal abaixo de 35\%.

Houve alta proporção de sobrepeso e obesidade nos policiais militares avaliados. A soma de policiais com diagnóstico de sobrepeso e obesidade foi de 117 (63,9\%). Apenas 22 (12,0\%) policiais apresentaram risco muito elevado de

Tabela 1. Descrição de indicadores antropométricos e alimentares de policiais militares. Cascavel (PR), 2006.

\begin{tabular}{|c|c|c|c|c|c|c|}
\hline Variáveis estudadas & $\mathrm{n}$ & Média & Mediana & Desvio-Padrão & \multicolumn{2}{|c|}{ Mínimo-Máximo } \\
\hline IMC $\left(\mathrm{Kg} / \mathrm{m}^{2}\right)$ & 183 & 26,6 & 26,2 & 4,0 & 19,80 & - $\quad 47,2$ \\
\hline Circunferência abdominal (cm) & 183 & 90,5 & 90,0 & 10,5 & 72,40 & $-136,0$ \\
\hline Gordura corporal (\%) calculado & 140 & 20,9 & 20,3 & 5,0 & 10,40 & $-\quad 42,5$ \\
\hline Gasto energético basal (kcal) & 183 & 738,0 & 1729,0 & 118,2 & 1424,00 & $-2054,0$ \\
\hline Ingestão energética (kcal) & 183 & 2230,0 & 2047,0 & 812,0 & 746,93 & $-4773,5$ \\
\hline Ingestão gordura total (g) & 75 & 31,0 & 22,7 & 33,8 & 75,00 & $-\quad 239,0$ \\
\hline Ingestão gordura total (\%) & 75 & 39,3 & 37,8 & 7,8 & 25,90 & $-\quad 67,0$ \\
\hline Ingestão gordura saturada (g) & 75 & 30,8 & 25,2 & 21,7 & 3,20 & $-\quad 110,5$ \\
\hline Ingestão gordura saturada (\%) & 75 & 9,3 & 7,7 & 6,7 & 1,40 & - $\quad 43,4$ \\
\hline
\end{tabular}

IMC: índice de massa corporal. 
complicações metabólicas associadas à obesidade, de acordo com o valor de CA, e 32 (21,3\%) apresentavam risco de doenças associadas à obesidade devido ao alto percentual de gordura corporal (Tabela 2).

Tabela 2. Estado nutricional de policiais militares, segundo o índice de massa corporal, a circunferência abdominal e o percentual de gordura corporal. Cascavel (PR), 2006.

\begin{tabular}{lcc}
\hline Dados antropométricos & $\mathrm{n}$ & $\%$ \\
\hline Estado nutricional baseado no IMC & 66 & 36,1 \\
Eutrófico & 83 & 45,4 \\
Sobrepeso & 30 & 16,4 \\
Obeso I & 1 & 0,5 \\
Obeso II & 3 & 1,6 \\
Obeso III & 183 & 100,0 \\
\hline Total & & \\
\hline Circunferência abdominal & 22 & 12 \\
Igual ou acima de 102cm & 161 & 88 \\
Abaixo de 102cm & 183 & 100,0 \\
\hline Total & \multicolumn{3}{l}{} \\
\hline Percentual de gordura corporal & 14 & 9,4 \\
Igual ou abaixo da média $\leq 15 \%$ & 104 & 69,3 \\
Acima da média 15,1-24,9\% & 32 & 21,3 \\
Igual ou acima de 25\% - Risco de doenças & \multicolumn{3}{l}{} \\
associadas à obesidade & 150 & 100,0 \\
\hline Total & &
\end{tabular}

Tabela 3. Avaliação da ingestão alimentar de lipídios totais e saturados de policiais militares. Cascavel (PR), 2006.

\begin{tabular}{lcc}
\hline Ingestão de lipídios & $\mathrm{n}$ & $\%$ \\
\hline Lipídios totais & & \\
Excessivo $(>35 \%)$ & 53 & 70,7 \\
Adequado $(\leq 35 \%)$ & 22 & 29,3 \\
\hline Total & 75 & 100 \\
\hline Lipídios saturados & & \\
Excessivo $(>10 \%)$ & 24 & 32 \\
Adequado $(\leq 10 \%)$ & 51 & 68 \\
\hline Total & 75 & 100 \\
\hline
\end{tabular}

Encontrou-se correlação positiva entre IMC, CA e percentual de gordura corporal. Quanto maior o IMC, maior a CA $(r=0,87, p<0,001)$ e maior o percentual de gordura corporal $(r=0,76$, $p<0,001)$. O percentual de gordura também mostrou correlação positiva com a $C A(r=0,76$, $p<0,001)$.

Observa-se, na Tabela 3, que 53 (70,7\%) dos indivíduos com inquéritos validados relataram ingerir dieta hiperlipídica. Com relação aos lipídios saturados, 24 policiais $(32,0 \%)$ consomem acima de $10,0 \%$, que é o máximo de ingestão preconizado para uma alimentação saudável.

Encontrou-se correlação positiva significante somente entre ingestão de lipídios totais e circunferência abdominal $(r=0,24 p=0,03)$ (Tabela 4).

\section{DIS C U S S Ã O}

Dos policiais avaliados, $83(45,4 \%)$ encontrava-se com sobrepeso e $34(18,5 \%)$ eram obesos. De acordo com a Pesquisa de Orçamentos Familiares, a prevalência de sobrepeso e obesidade em homens na zona urbana da região sul do Brasil é de $47,7 \%$ e $10,7 \%{ }^{5}$, respectivamente. Segundo os dados da Vigitel Brasil ${ }^{19}$, em Curitiba, a prevalência de sobrepeso em homens foi de 49,8\% e de obesidade, 12,9\%. Observa-se que a proporção de obesidade apresentada pelos policiais militares se encontra acima da média nesta Região.

A obesidade é determinada pela combinação de predisposição genética, de desequilíbrios no balanço energético e de fatores ambientais e sociais $^{20,21}$. Estima-se que entre $40 \%$ a $70 \%$ da variação no fenótipo associado à obesidade apresenta caráter hereditário e envolve fatores tais

Tabela 4. Correlação entre a ingestão de lipídios e os indicadores antropométricos de adiposidade de policiais militares. Cascavel (PR), 2006.

\begin{tabular}{|c|c|c|c|c|c|c|}
\hline & \multicolumn{3}{|c|}{ Ingestão de lipídios totais } & \multicolumn{3}{|c|}{ Ingestão de lipídios saturados } \\
\hline & r & $p$ & $\mathrm{n}$ & r & $p$ & $n$ \\
\hline Índice de massa corporal & 0,20 & 0,089 & 75 & 0,16 & 0,150 & 75 \\
\hline Circunferência abdominal & 0,24 & 0,034 & 75 & 0,11 & 0,320 & 75 \\
\hline Percentual de gordura corporal & 0,19 & 0,148 & 57 & 0,15 & 0,250 & 57 \\
\hline
\end{tabular}


como alterações no apetite ou gasto energético, sendo estas determinações genéticas importantes para determinar a distribuição de gordura corpórea, influenciando especialmente na predisposição de depósito de gordura visceral ${ }^{21}$.

Não se encontrou alto índice de aumento da circunferência abdominal entre os policiais avaliados. Sabe-se que a circunferência abdominal isoladamente vem mostrando boa correlação com a quantidade de gordura abdominal associada ao processo saúde-doença, sendo que as alterações observadas na CA são consideradas preditores de risco para doenças cardiovasculares ${ }^{22,23}$. Martins \& Marinho ${ }^{24}$, em estudo de amostra da população do Município de São Paulo, analisaram a relação entre dislipidemia, IMC e CA, verificando que a CA relacionou-se com a hipercolesterolemia, favorecendo a hipótese de que a circunferência abdominal é indicadora de risco doença cardiovascular aterosclerótica. Ainda com relação à distribuição central de gordura, vários estudos $20,25,26$ encontraram relação positiva com a prevalência de hipertensão, demonstrando associação entre gordura abdominal e aumento da prevalência de outros fatores de risco para doenças cardiovasculares.

Encontrou-se correlação positiva entre IMC, CA e percentual de gordura corporal nos policiais militares. Em estudos com amostras representativas da população americana e de outros países desenvolvidos, demonstrou-se que o IMC correlaciona-se significativamente com a massa de gordura corporal, com o percentual de gordura corporal, e também com a circunferência abdominal27.

Embora o presente estudo tenha verificado correlação positiva entre o IMC e o percentual de gordura corporal, houve baixa proporção de policiais com alta concentração de gordura corporal. Correia et al..$^{28}$ e Junior et al. ${ }^{29}$ estudaram a influência da gordura corporal no controle clínico e metabólico de pacientes diabéticos, encontrando associação positiva entre a gordura corporal e a circunferência da cintura, sugerindo possível correlação com a síndrome metabólica.
Encontrou-se alto índice de sub-relato da ingestão alimentar nesta população estudada, 108 (59\%) policiais relataram ingerir quantidade bem menor que suas necessidades energéticas. Este fato pode ter ocorrido devido ao elevado número de indivíduos com sobrepeso e obesidade, pois indivíduos com excesso de peso tendem a subestimar sua ingestão energética. Outro fator que pode estar associado ao sub-relato é a não atenção à quantidade de alimentos ingeridos. É importante ressaltar alguns fatores que dificultaram a realização das entrevistas dos policiais militares, como a falta de disponibilidade de horário, o cansaço após a jornada de trabalho, escalas de plantões noturnos, contribuindo assim para a irregularidade na alimentação, entre outros fatores.

A avaliação dos 75 inquéritos validados mostrou que a maioria dos indivíduos consome excessiva quantidade de lipídios, porém com baixo índice de policiais ultrapassando 10,0\% de gordura saturada. Este resultado constata que a qualidade destes lipídios encontra-se dentro do padrão aceitável para indivíduos sadios. Segundo a Pesquisa de Orçamentos Familiares, a população da zona urbana na Região Sul do Brasil apresenta consumo médio de $32,0 \%$ de lipídios totais em sua dieta, sendo que, deste total, 9,9\% são provenientes de lipídios saturados ${ }^{5}$. A ingestão média de lipídios totais dos policiais militares foi de 39,3\% (131g) e de lipídios saturados 9,3\% $(30,8 \mathrm{~g})$, chamando a atenção, portanto, apenas para o excesso de lipídios totais.

Cervato et al..$^{30}$ estudaram 557 homens e mulheres, entre 20 e 40 anos, analisando riscos na dieta habitual para doenças cardiovasculares, e observaram que o consumo médio de lipídios foi de, aproximadamente, 64g, representando 29\% da contribuição energética, porém, para 25\% da população, a proporção para os lipídios estava maior que $34 \%$, sendo que $25 \%$ da população ingeriam dietas aterogênicas, com alto teor de lipídios e ácidos graxos saturados.

Os ácidos graxos saturados de cadeia longa estão presentes em maior concentração nas gordu- 
ras alimentares de origem animal. Existe forte associação entre o consumo de gorduras saturadas e a aterosclerose, principalmente devido ao aumento dos níveis plasmáticos de colesterol e Lipoproteínas de Baixa Densidade (LDL-C) ${ }^{31}$.

Não se encontrou, no presente estudo, correlação entre ingestão de lipídios saturados e os índices antropométricos avaliados: IMC, CA e percentual de gordura corporal, sugerindo que este tipo de gordura não teve influência nos indicadores antropométricos estudados. Entretanto, houve relação positiva entre ingestão de gorduras totais e circunferência abdominal, que merece aprofundamento. Embora em uma população diferenciada, estudo que avaliou a associação entre consumo alimentar e presença de obesidade abdominal em indivíduos infectados pelo HIV/AIDS, encontrou resultados que sugerem que a quantidade de carboidratos e lipídios na dieta, independentemente do consumo energético, pode modificar a chance de desenvolver obesidade abdominal na população estudada ${ }^{32}$. Não foram encontrados na literatura outros estudos que relacionassem ingestão lipídica e circunferência abdominal ou percentual de gordura corporal.

A utilização de apenas dois recordatórios de 24 horas pode ser considerada uma limitação deste estudo, pois a literatura sugere a realização de três ou mais recordatórios para redução da variabilidade intra-individual ${ }^{33,34}$. Por esse motivo, incluiu-se a validação dos inquéritos por meio do sub-relato de ingestão, como forma de minimizar erros na avaliação da ingestão energética e lipídica.

Em conclusão, este estudo sugere que a ingestão de lipídios acima das recomendações é um fator que pode estar relacionado à adiposidade abdominal na população estudada. Ações nutricionais, como a educação e o aconselhamento nutricional, o tratamento das co-morbidades voltadas para esta população, bem como, a melhora na oferta da alimentação fornecida durante a jornada de trabalho, pode contribuir positivamente para a melhora dos hábitos alimentares, prevenindo o aparecimento de doenças crônicas não-transmissíveis e melhorando a qualidade de vida destes policiais.

\section{A GRADECIMENTOS}

Ao Tenente Coronel Nelson João Casarolli e ao subcomandante Major Celso Luis Borges, atual comandante do batalhão, e ao cabo da equipe do $6^{\circ}$ BPM, pela disponibilidade na participação, e ao Cabo José Gilberto Viana Bones, que nos auxiliou na organização das coletas em campo.

\section{COLABORADORES}

C. DONADUSSI auxiliou nas coletas antropométricas e alimentares, analisou os dados específicos e elaborou este artigo. A.F. OLIVEIRA e E.C.S. FATEL responsáveis pela coleta de dados, auxiliou na elaboração e na revisão deste artigo. J.B. DICHI coordenadora do projeto e revisora final deste artigo. I. DICHI revisor final deste artigo.

\section{REFERÊ NCIAS}

1. Brasil. Ministério da Saúde. A vigilância, o controle e a prevenção das doenças crônicas não-transmissíveis: DCNT no contexto do Sistema Único de Saúde brasileiro/Brasil. Brasília: Ministério da Saúde; 2005.

2. Dehoog S. Avaliação do estado nutricional. In: Mahan K, Escott S. Krause alimentos, nutrição e dietoterapia. 10a. ed. São Paulo: Roca; 2002.

3. Francischi RPP, Pereira LO, Freitas CS, Klopfer M, Santos RC, Lancha Junior AH. Obesidade: atualização sobre sua etiologia, morbidade e tratamento. Rev Nutr. 2000; 13(1):17-28. doi: 10.1590/ S1415-52732000000100003.

4. Camacho LAB, Ell E, Chor D. Perfil antropométrico de funcionários de banco estatal no estado do Rio de Janeiro/Brasil: índice de massa corporal e fatores sócio-demográficos. Cad Saúde Pública. 1999; 15(1):113-22. doi: 10.1590/S0102-311X1999000 100012.

5. Instituto Brasileiro de Geografia e Estatística. Pesquisa de Orçamento Familiares 2002-2003: análise da disponibilidade domiciliar de alimentos e do estado nutricional do Brasil. Rio de Janeiro: IBGE; 2004.

6. Schneider HJ, Glaesmer $\mathrm{H}$, Klotsche J, Böhler S, Lehnert $H$, Zeither AM, et al. Accuracy of anthropometric indicators of obesity to predict cardiovascular risk. J. Clin End Metab. 2004; 92 (2):589-94. doi:10.1210/jc.2006-0254. 
7. Lima FEL, Menezes TN, Tavares MP, Szarfarc SC, Fisberg RM. Ácidos graxos e doenças cardiovasculares: uma revisão. Rev Nutr. 2000; 13(2):73-80. doi: 10.1590/S1415-52732000000200001.

8. World Health Organization. Physical Status: the use and interpretation of anthropometry. Geneva; WHO; 1995. Report of a WHO Expert Committee.

9. Brasil. Ministério da Saúde. Vigilância alimentar e nutricional. Orientações básicas para a coleta, análise de dados e a informação em serviços de saúde. Brasília; 2004.

10. World Health Organization. Obesity: preventing and managing the global epidemic. Geneva: WHO; 1998. Report of a WHO Consultation on Obesity.

11. Expert Panel on Detection, Evaluation and Treatment of High Blood Cholesterol in Adults. Executive summary of the Third Report of the National Cholesterol Education Program (NCEP) Expert Panel on Detection, Evaluation and Treatment of High Cholesterol. JAMA 2001; 285(19): 2486-97. doi:10.1234/12345678.

12. Lohman TG, Roche AF, Martorell R. Anthropometric standardization reference manual. Champaign: Human Kinetics Books; 1991.

13. Costa AGV, Priore SE, Sabaraense CM, Franceschini SCC. Questionário de freqüência de consumo alimentar e recordatório de 24 horas: aspectos metodológicos para avaliação da ingestão de lipídios. Rev Nutr. 2006; 19(5):631-641. doi: 10.1590/S1415-52732006000500011.

14. NUT: Sistema de Apoio e Decisão em Nutrição [programa de computador]. Versão 2,5a. Departamento de Informática em Saúde. São Paulo: Unifesp-EPM; 1998.

15. Institute of Medicine. Dietary references intakes for energy, carbohydrate, fiber, fat, fatty acids, cholesterol, protein and aminoacids (macronutrients). Washington (DC): National Academy Press; 2005.

16. Avesani CM, Santos NSJ, Cuppari L. Necessidades e recomendações de energia. In: Cuppari L. Guia de nutrição: nutrição clínica no adulto. Barueri: Manole; 2005.

17. Goldberg GR, Black AE, Jebb AS, Cole TJ, Murgatroyd PR, Coward WA, et al. Critical evaluation of energy intake data using fundamental principles of energy intake physiology: 1. Derivation of cut-off limits to identify under-recording. Eur J Clin Nutr. 1991; 45(12): 569-81.
18. National Research Council. Recommended dietary allowances. Washington (DC): National Academy Press; 1989.

19. Brasil. Ministério da Saúde. Vigitel Brasil 2007. Vigilância de fatores de risco e proteção para doenças crônicas por inquérito telefônico. Série G. Estatística e informação à saúde. Brasília; 2008.

20. Shrestha J. Clinical study of overweight individuals in correlation to type-2 diabetes mellitus, hyperension and hyperlipidaemia [tese]. Kathmandu (Nepal): Internal Medicine of Tribhuvan University; 2004.

21. Lopes-Marques I, Marti A, Moreno-Aliaga MJ, Martínez A. Aspectos genéticos da obesidade. Rev Nutr. 2004; 17(3):327-38. doi: 10.1590/S141552732004000300006.

22. Castro LCV, Franceschini SCC, Priori SE, Pelúzio MCG. Nutrição e doenças cardiovasculares: os marcadores de risco em adultos. Rev Nutr. 2004; 17(3):369-77. doi: 10.1590/\$1415-52732004000 300010 .

23. Klein S, Allison DB, Heymsfield SB, Kelley DE, Leibel $\mathrm{RL}$, Nonas C, et al. Waist circumference and cardiometabolic risk: a consensus statement from Shaping America's Health. Am J Nutr. 2007; 85(5): 1197-202. doi: 10.2337/dc07-9921.

24. Martins IS, Marinho SP. O potencial diagnóstico dos indicadores da obesidade centralizada. Rev Saúde Pública. 2003; 37(6):760-67. doi: 10.1590/ S0034-89102003000600011.

25. Guedes DP, Guedes JERP. Distribuição de gordura corporal, pressão arterial e níveis de lipídiosproteínas plasmáticas. Arq Bras Cardiol. 1998; 70(2):93-8. doi: 10.1590/S0004-273020060006 00009 .

26. Carneiro G, Faria NA, Filho FFR, Guimarães A, Lerário D, Ferreira SRG, et al. Influência da distribuição da gordura corporal sobre a prevalência de hipertensão arterial e outros fatores de risco cardiovascular em indivíduos obesos. Rev Assoc Med Bras. 2002; 49(3):306-11. doi: 10.1590/S0 104-42302003000300036.

27. Anjos LA. Índice de massa corporal como indicador do estado nutricional de adultos: revisão da literatura. Rev Saúde Pública. 1992; 26(6):431-6. doi: 10.1590/S0034-89101992000600009.

28. Corrêa FHS, Taboada GF, Júnior CRMA, Faria AM, Clemente ELS, Fuks AG, et al. Influência da gordura corporal no controle clínico e metabólico de pacientes com diabetes mellitus tipo 2. Arq Bras Endocrinol Metab. 2003; 47(1):62-8. doi: 10.1590/ S0004-27302003000100010. 
29. Junior ACRM, Clemente EL, Gomes MB. Influência da gordura corporal em parâmetros de controle clínico e metabólico de pacientes com diabetes mellitus tipo 1. Arq Bras Endocrinol Metab. 2004; 48(6):885-89. doi: 10.1590/S0004-27302004000 600016 .

30. Cervato AM, Mazzilli RN, Martins IS, Marucci MFN. Dieta habitual de risco para doenças cardiovasculares. Rev Saúde Pública. 1997; 31(3):227-35. doi: 10.1590/S0034-89101997000300003.

31. National Cholesterol Education Program. Third Report of the National Cholesterol Education Program (NCEP) Expert Panel on Detection, Evaluation, and Treatment of High Blood Cholesterol in Adults (Adult Treatment Panel III). Final Report. Washington (DC): National Institutes of Health; 2002.
32. Constante JP, Antonio FA, Oliveira LMRD, Cotrim SAA. Central obesity and dietary intake in HIVIAIDS patients. Rev Saúde Pública. 2006; 40(4):634-40. doi: 10.1590/S0034-89102006000500012.

33. Fisberg RM, Martini LA, Slater BM. Métodos de inquéritos alimentares. In: Fisberg RM, Slater BM, Marchioni DML, Martini LA. Inquéritos alimentares: métodos e bases científicos. Barueri: Manole; 2005.

34. Willet WC. Nutritional epidemiology. 2nd ed. Oxford: Oxford University Press; 1998.

Recebido em: 10/12/2007

Versão final reapresentada em: 12/05/2009

Aprovado em: 6/10/2009 